Artículo científico

(Original paper)

\title{
DIVERSIDAD Y CONSERVACIÓN DE ANFIBIOS Y REPTILES EN UN ÁREA PROTEGIDA PRIVADA DE UNA REGIÓN ALTAMENTE TRANSFORMADA EN EL SUR DE VERACRUZ, MÉXICO
}

\author{
AMPHIBIAN AND REPTILE CONSERVATION IN A PRIVATELY PROTECTED AREA \\ FROM A HIGHLY TRANSFORMED REGION IN SOUTHERN VERACRUZ, MEXICO
}

\author{
José Luis AGUILAR-LÓPEZ ${ }^{1}$, LILIANA ORTIZ-LOZADA", JAIME PELAYO-MARTÍNEZ², ClaUdio \\ MOTA-VARGAS ${ }^{1}$, LUIS ERNESTO ALARCÓN-VILLEGAS ${ }^{2}$, ANA PAULINA DEMENEGHI-CALATAYUD ${ }^{3}$ \\ ${ }^{1}$ Instituto de Ecología, A.C., Carretera antigua a Coatepec 351, El Haya, C.P. 91073, Xalapa, Veracruz, México. \\ <jlal.herp@gmail.com>; <claudio.mota@inecol.mx> \\ ${ }^{2}$ Servicios Especializados en Estudios Integrales Ambientales, Argentina 35, C.P. 91070, Xalapa, Veracruz, México. \\ <bioliliana@gmail.com>; <casadecarrizo@hotmail.com>; <s_koatl@hotmail.com> \\ ${ }^{3}$ Braskem Idesa, Complejo Petroquímico, Nanchital, C.P. 96400, Veracruz, México. <paulina.demeneghi@braskem.com> \\ *Autor corresponsal: <bioliliana@gmail.com>
}

Recibido: 11/12/2018; aceptado: 30/01/2020; publicado en línea: 25/03/2020 Editor responsable: Eduardo Pineda

Aguilar-López, J. L., Ortiz-Lozada, L., Pelayo-Martínez, J., Mota-Vargas, C., Alarcón-Villegas, L. E., Demeneghi-Calatayud, A. P. (2020) Diversidad y conservación de anfibios y reptiles en un área protegida privada de una región altamente transformada en el sur de Veracruz, México. Acta Zoológica Mexicana (nueva serie), 36, 1-14. https://doi.org/10.21829/azm.2020.3612164

RESUMEN. El establecimiento de Áreas Protegidas Privadas (APPs) es una de las estrategias para la protección de la biodiversidad ante la modificación del hábitat en bosques tropicales; sin embargo, su importancia en conservación ha sido poco evaluada. En este trabajo estimamos la importancia de un APP conocida como Área de Protección y Desarrollo de Ceratozamia (APDC) para la conservación de anfibios y reptiles, en comparación con otras dos APPs (Parque Ecológico Jaguaroundi y el Parque Ecológico Tuzandepetl) en el sur de Veracruz, México. El APDC tiene una extensión de 100 hectáreas: 50 ha de bosque tropical subcaducifolio y 50 ha de pastizal. Los muestreos para este estudio se realizaron entre los años 2015 y 2016, empleando el método de búsqueda libre restringida por tiempo (esfuerzo de muestreo total $=768$ horas-persona). En total, en el APDC se registraron 20 especies de anfibios y 35 de reptiles. El 20\% de los anfibios y el 32\% de reptiles registrados se encuentran en alguna categoría de riesgo de extinción según la Norma Oficial Mexicana NOM-059-SEMARNAT-2010, dos especies de anfibios y una especie de reptil están incluidas en la Lista Roja de la Unión Internacional para la Conservación de la Naturaleza (UICN), mientras que el $12 \%$ de los reptiles se encuentran en categoría de vulnerabilidad ambiental alta. El APDC tuvo la mayor riqueza de especies en comparación con las otras dos APPs. Los anfibios tuvieron una composición relativamente similar entre las APPs, mientras que para los reptiles la composición de especies tendió a ser distinta. Nuestros resultados indican que por la riqueza de especies y el número de especies amenazadas de extinción y de especies no evaluadas por la UICN que alberga, el APDC contribuye a la conservación de herpetozoos en una zona altamente transformada del sur de México, en conjunto con las otras dos APPs, donde el Área Natural Protegida bajo régimen gubernamental más cercana (Reserva de la Biosfera Los Tuxtlas) se localiza a $50 \mathrm{~km}$. 
Palabras clave: anfibios; reptiles; composición de especies; reemplazo de especies; especies amenazadas; bosque tropical

Aguilar-López, J. L., Ortiz-Lozada, L., Pelayo-Martínez, J., Mota-Vargas, C., Alarcón-Villegas, L. E., Demeneghi-Calatayud, A. P. (2020) Amphibian and reptile conservation in a privately protected area from a highly transformed region in southern Veracruz, Mexico. Acta Zoológica Mexicana (nueva serie), 36, 1-14. https://doi.org/10.21829/azm.2020.3612164

\begin{abstract}
The establishment of Private Protected Areas (PPAs) is one of the strategies for the protection of biodiversity in face to habitat modification in tropical forests; however, its importance in conservation has been poorly evaluated. In this work we estimate the importance of a PPA, known as Área de Protección y Desarrollo de Ceratozamia (APDC) for the conservation of amphibians and reptiles, compared to two other PPAs (Parque Ecológico Jaguaroundi and Parque Ecológico Tuzandepetl) at southern Veracruz, Mexico. The APDC has an area of 100 hectares: 50 ha of tropical deciduous forest and 50 ha of cattlepasture. The surveys for this study were carried out between 2015 and 2016, which consisted of free search restricted by time (total sampling effort $=768$ person-hours). In total, 20 species of amphibians and 35 species of reptiles were recorded in the APDC. Twenty percent of amphibians and $32 \%$ of registered reptiles are in some extinction risk category according to the Norma Oficial Mexicana NOM-059-SEMARNAT2010, two amphibian species and one reptile species are included in the Red List of the International Union Conservation of Nature (IUCN), while $12 \%$ of reptiles are in high category of environmental vulnerability. The APDC has the highest species richness in comparison to the other two PPAs. Amphibians had a relatively similar composition among the PPAs, while for reptiles, species composition tended to be relatively different. Our results indicate that due to the richness of the species, the number of endangered species and species not evaluated by IUCN it harbors, the APDC contributes to the conservation of herpetozoa in a highly transformed area of southern Mexico, together with the two other PPAs, where the closest Protected Natural Area under governmental regime (Los Tuxtlas Biosphere Reserve) is located 50 km away.
\end{abstract}

Key words: amphibians; reptiles; species composition; species replacement; threatened species; evergreen seasonal forest

\title{
INTRODUCCIÓN
}

Los bosques tropicales son ecosistemas que contienen una alta biodiversidad, pero también se encuentran sometidos a una rápida y severa transformación por las distintas actividades humanas, como la agricultura, la ganadería extensiva, la industria y el crecimiento de asentamientos humanos (Hansen et al., 2013; Sánchez-Colón et al., 2009). En el sur de México, este proceso de modificación de cambio de uso de suelo ha generado paisajes compuestos por pequeños fragmentos de hábitat original, inmersos en una matriz de ambientes modificados (Sommer \& Oropeza-Orozco, 2010; Schmitter-Soto et al., 2016). Ante esta problemática, una de las estrategias de conservación más difundidas para la protección de la biodiversidad ha sido el establecimiento de Áreas Naturales Protegidas (ANPs; Naughton-Treves et al., 2005).

Las ANPs terrestres en México pueden ser clasificadas, de acuerdo con su tipo de gobernanza, en tres categorías: las de carácter gubernamental, que pueden ser municipales, estatales o federales; las de carácter comunitario, las cuales son manejadas comúnmente por comunidades rurales o ejidos; y las de carácter privado, que son conocidas como Áreas Protegidas Privadas (APPs). Éstas últimas son definidas por la Unión Internacional para la Conservación de la Naturaleza (IUCN, por sus siglas en inglés) como "un espacio geográfico claramente definido, reconocido, dedicado y manejado, a través de medios legales u 
otros medios efectivos, para lograr la conservación a largo plazo de la naturaleza, bajo gobernanza privada, ya sea de individuos o instituciones" (Stolton et al., 2014).

Dado que el número de APPs en México es considerable, al menos 407 certificadas por instituciones gubernamentales y 285 no certificadas (Bezaury-Creel, 2014), su contribución para la conservación de la biodiversidad en el escenario actual de transformación de la cobertura vegetal en el país, podría ser significativa; sin embargo, dicha contribución ha sido poco evaluada (Cruz-Bazán et al., 2017). Entre las primeras tareas necesarias para determinar el aporte a la conservación de la biodiversidad de un APP, está examinar la diversidad biológica que alberga y evaluar el número de especies amenazadas que contiene. Además, se puede comparar su diversidad de especies con la de áreas protegidas cercanas para evaluar su complementariedad. Con base en esta información, se pueden generar planes de manejo y conservación para distintos grupos taxonómicos, adecuados a las características del APP y las condiciones del paisaje circundante.

Los anfibios y reptiles son componentes clave de los ecosistemas, ya que son importantes consumidores de insectos y se encuentran entre las presas más consumidas por otros grupos de vertebrados, como aves, mamíferos y de otros reptiles (Cortés-Gómez et al., 2015). Estos dos grupos biológicos se encuentran amenazados a nivel global, debido principalmente a la destrucción o modificación de su hábitat (Wake, 1991; Böhm et al., 2013). En México, la mitad de las especies de anfibios y una décima parte de los reptiles se encuentran en alguna categoría de amenaza de la Lista Roja de la Unión Internacional para la Conservación de la Naturaleza [UICN] (IUCN, 2017) o de la lista de especies en riesgo de la Norma Oficial Mexicana NOM-059-SEMARNAT-2010 (SEMARNAT, 2010). No obstante, el sistema de áreas naturales protegidas gubernamentales en México no garantiza la protección de todos los anfibios y reptiles del país (Urbina-Cardona \& Flores-Villela, 2010; Ochoa-Ochoa et al., 2009), por lo que resulta necesario explorar la contribución de las APPs para la conservación de estos grupos biológicos (Cruz-Elizalde et al., 2015).

Como parte de los esfuerzos para la conservación de la biodiversidad de los bosques tropicales en el sur de México, en 1998 se estableció la Reserva de la Biosfera Los Tuxtlas, bajo administración federal (CONANP, 2006). A partir de esa fecha, una serie de áreas naturales de carácter privado se han establecido en el sur de México, como el Parque Ecológico Jaguaroundi (PEJ, Herrera et al., 2008) creado en 1998, el Parque Ecológico Tuzandepetl (PET, Cruz-Silva, 2012) creado en 1999 y el Área de Protección y Desarrollo de Ceratozamia (APDC) creada en el año 2015 (SEDEMA, 2015). En las dos primeras APPs se ha examinado ya la riqueza y la composición de especies de herpetofauna que contienen (Herrera et al., 2008; Cruz-Silva, 2012) pero de la última no se tiene conocimiento sobre los anfibios y reptiles que ahí habitan.

El objetivo de este estudio fue evaluar la importancia del Área de Protección y Desarrollo de Ceratozamia, ubicada en una zona altamente transformada de bosque tropical del sur de México, para la conservación de anfibios y reptiles, considerando el número e identidad de las especies, así como su estado de conservación de acuerdo con la NOM-059-SEMARNAT-2010, la Lista Roja de la UICN y el Puntaje de Vulnerabilidad Ambiental. Adicionalmente, se examinó la contribución complementaria del APCD (diferencia en composición de especies) con respecto a otras dos áreas protegidas privadas, localizadas en la misma zona, y al área natural protegida de carácter gubernamental más próxima.

\section{MATERIALES Y MÉTODOS}

Área de estudio. El estudio se realizó en el Área Protegida Privada denominada Área de Protección y Desarrollo de Ceratozamia (APDC), localizada en el Municipio de Ixhuatlán del Sureste, estado de Veracruz, en el sur de México (Fig. 1). El área tiene una altitud promedio de $60 \mathrm{~m}$, una precipitación media anual de $1,800 \mathrm{~mm}$ y una temperatura media anual de $27^{\circ} \mathrm{C}$. El tipo de vegetación original en la zona corresponde a bosque tropical subcaducifolio (Castillo-Campos et al., 2011). El APDC tiene una superficie 
de 100 hectáreas, de las cuales 50 ha corresponden a un área abierta dominada por pasto inducido para la ganadería y otras 50 ha corresponden a bosque tropical subcaducifolio conservado. La zona de pastizal está dominada por pastos inducidos para la ganadería (Paspalum fasciculatum y Setaria geniculata), con algunos árboles aislados (Coccoloba hondurencis), algunas palmas (Attalea liebmannii) y con cercas vivas compuestas por C. hondurencis, Guazuma ulmifolia, Bursera simaruba y Gliciridia sepium. El área de remanentes de bosque tropical subcaducifolio tiene un estrato arbóreo con una altura máxima de $25 \mathrm{~m}$, donde las especies más representativas son Miconia argentea, Guazuma ulmifolia, Cupania dentata, $C$. hondurencis, Bursera simaruba, Enterolobium cyclocarpum, Cecropia obtusifolia, Vochysia hondurensis, Pithecellobium lanceolatum. En el estrato arbóreo bajo, las especies dominantes son Tabernaemontana alba y Dendropanax arboreus.

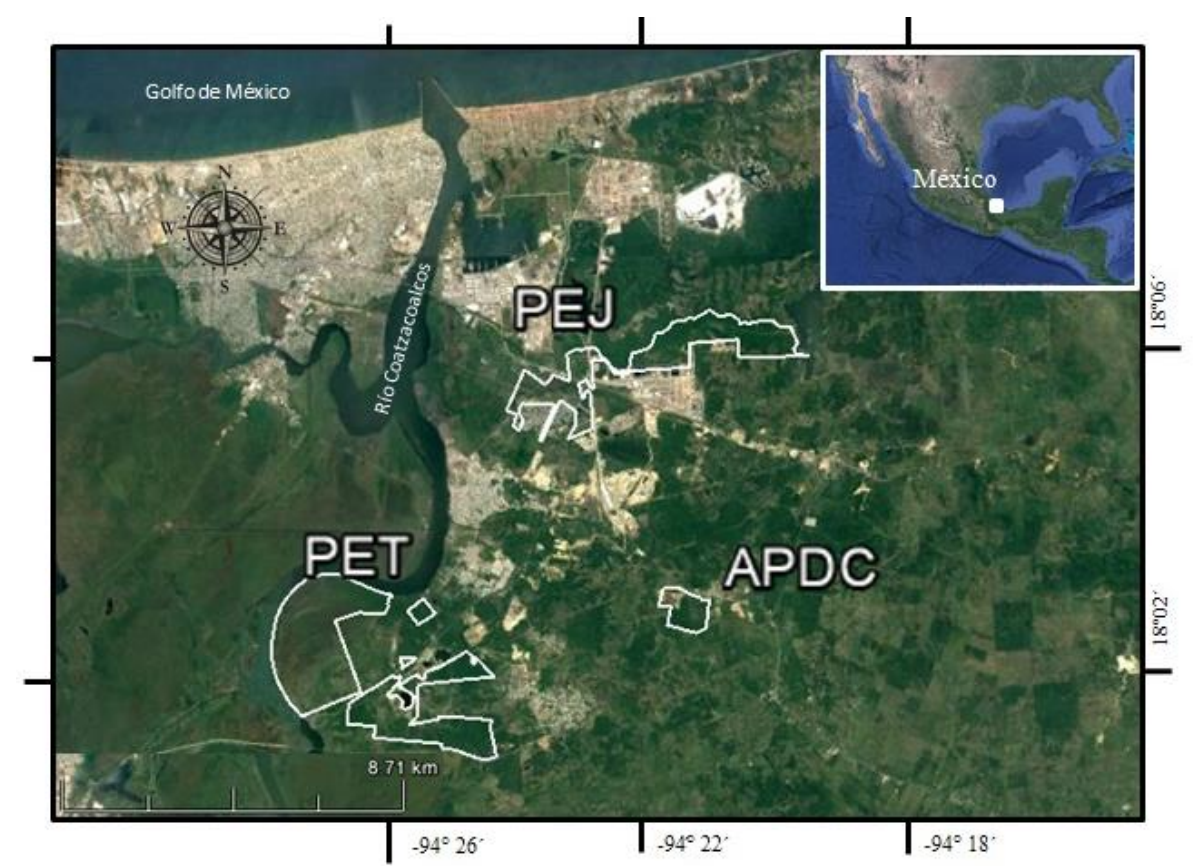

Figura 1. Ubicación del Área de Protección y Desarrollo de Ceratozamia (APDC), el Parque Ecológico Jaguaroundi (PEJ) y el Parque Ecológico Tuzandepetl (PET), en el sur de Veracruz, México. Las líneas blancas representan los polígonos de cada una de las áreas protegidas.

Trabajo de campo. Los muestreos se realizaron durante ocho días en tres épocas del año: temporada de secas (abril), temporada de lluvias (julio) y temporada de nortes (diciembre), durante 2015 y 2016, tanto en bosque tropical conservado como en pastizal, para detectar el mayor número de especies posible. Los muestreos fueron realizados por dos personas, empleando las técnicas de búsqueda libre restringida por tiempo (Crump \& Scott, 1994), tanto en horario diurno de 06:30-10:30 horas, como vespertino-nocturno de 16:00-20:00 horas. El esfuerzo de muestreo por tipo de uso de suelo fue de 384 horas-persona, dando como resultado un esfuerzo total de muestreo de 768 horas-persona. Todos los individuos capturados fueron registrados, fotografiados e identificados con guías de campo (Köhler, 2008; 2011) para posteriormente ser liberados en el mismo sitio de su captura. La nomenclatura de las especies de anfibios se basó en Amphibian Species of the World 6.0 (Frost, 2017) y en el caso de los reptiles se consultó Reptile Database (Uetz et al., 2018). El estado de conservación de cada una de las especies registradas se determinó con base en la Norma Oficial Mexicana NOM-059-SEMARNAT-2010 (SEMARNAT, 2010), la Lista Roja de la UICN (2017) y en el Puntaje de Vulnerabilidad Ambiental (EVS= Environmental Vulnerability Score) propuesto por Wilson et al. (2013a; b), el cual considera la extensión geográfica, la extensión de la distribución ecológica (tipo de vegetación) y el modo reproductivo (en anfibios) o el grado de amenaza por el humano (en reptiles). 
Las categorías de vulnerabilidad son baja (B: de 3 a 9 puntos), media (M: de 10 a 13 puntos) y alta (A: de 14 a 20 puntos).

Análisis de datos. Para determinar el grado de completitud del inventario en el APDC se estimó la riqueza esperada, calculada con los estimadores no paramétricos Chao 2 y Jacknife de primer orden y de segundo orden (Moreno, 2001), los cuales se basan en datos de presencia-ausencia agrupados en muestras. Para ello se empleó el programa EstimateS versión 9.1 (Colwell, 2013).

Se determinaron las diferencias en composición de especies entre el APDC y otras dos APPs, el Parque Ecológico Jaguaroundi (PEJ, Herrera et al., 2008) y el Parque Ecológico Tuzandepetl (PET, CruzSilva, 2012) ubicadas muy cerca geográficamente (distancia máxima entre áreas de $6.5 \mathrm{~km}$ en línea recta), en el sur de Veracruz (Fig. 1). Para esto se empleó la propuesta de Carvalho et al. (2012), en la cual se calcula la diferencia total en composición $\left(\mathrm{B}_{\mathrm{cc}}\right)$ de especies entre dos sitios o entidades, con la fórmula:

$$
\mathrm{B}_{c c}=\frac{b+c}{a+b+c}
$$

la proporción de la diferencia en composición debida al reemplazo de especies ( $\left.\mathrm{B}_{-3}\right)$ :

$$
\mathrm{B}_{-3}=2 \times \frac{\min (b, c)}{a+b+c}
$$

y la proporción de la disimilitud debida a la diferencia en riqueza de especies $\left(\mathrm{B}_{\text {rich }}\right)$ :

$$
B_{\text {rich }}=\frac{|b-c|}{a+b+c}
$$

En todas las fórmulas, $a$ es el número de especies presentes en ambos sitios, $b$ el número de especies exclusivamente en el primer sitio y $c$ el número de especies presentes exclusivamente en el segundo sitio. La suma de los valores derivados del reemplazo de especies $\left(\mathrm{B}_{-3}\right)$ y de la diferencia en riqueza de especies da como resultado la diferencia total en composición $\left(\mathrm{B}_{\mathrm{cc}}\right)$. Los valores obtenidos de $\mathrm{B}_{\mathrm{cc}}$ van de 0 (comunidades compuestas por las mismas especies) a 1 (comunidades compuestas por especies totalmente distintas). La especie Hemidactylus frenatus no fue considerada en este análisis por ser una especie exótica.

\section{RESULTADOS}

En el APDC se registraron 20 especies de anfibios (tres de salamandras y 17 de anuros) y 35 de reptiles (13 lagartijas, 16 serpientes y seis tortugas; Cuadro 1). De las 20 especies de anfibios, dos se encontraron exclusivamente en el bosque conservado, siete exclusivamente en el pastizal y 11 en ambos ambientes; mientras que, de las 35 especies de reptiles, 15 se registraron exclusivamente en el bosque tropical subcaducifolio, 11 exclusivamente en el pastizal y nueve en ambos ambientes (Cuadro 1). La riqueza estimada indica que el nivel del inventario para anfibios se encuentra entre el 91 y 100\% de completitud y que es probable que falten por registrar dos especies; mientras que, en el caso de los reptiles, el inventario se encuentra entre el 70 y $79 \%$ de completitud, faltando por registrar, entre nueve y 15 especies (Cuadro 2).

De las especies registradas, cuatro de anfibios y 11 de reptiles se encuentran incluidas en alguna categoría de riesgo de la NOM-059-SEMARNAT-2010, mientras que dos especies de anfibios y una de reptil están incluidas en categorías altas de riesgo de extinción según la UICN. En cuanto a las especies que no han sido evaluadas por la UICN encontramos una de anfibio y 20 de reptiles (Cuadro 1). Con respecto al Puntaje de Vulnerabilidad Ambiental, 13 especies de anfibios se encuentran en categoría de vulnerabilidad baja (B) y siete especies en vulnerabilidad media (M). En el caso de los reptiles, 16 se consideran en vulnerabilidad baja (B), 10 en vulnerabilidad media (M) y cuatro en vulnerabilidad alta (A), mientras que tres especies no tienen asignado un puntaje de vulnerabilidad (Cuadro 1). 
Cuadro 1. Especies de anfibios y de reptiles registradas en el Área de Protección y Desarrollo de Ceratozamia (APDC); (BTS = Bosque Tropical Subcaducifolio, PAS = Pastizal para ganado) y su estado de conservación según la NOM059-SEMARNAT-2010 (NOM-059; PR = Sujeta a protección especial, A = Amenazada), la Lista Roja de la Unión Internacional para la Conservación de la Naturaleza $(\mathrm{UICN}$; LC $=$ Least Concern o Preocupación Menor, VU = Vulnerable o Vulnerable, NT $=$ Near Threatened o Casi Amenazada, NE $=$ No Evaluada) y el Puntaje de Vulnerabilidad Ambiental (EVS) y categoría de vulnerabilidad ( $\mathrm{B}=$ baja, $\mathrm{M}=$ media y $\mathrm{A}=$ alta).

\begin{tabular}{|c|c|c|c|c|c|c|c|}
\hline \multirow{2}{*}{$\begin{array}{l}\text { Clase } \\
\text { Orden } \\
\text { Suborden }\end{array}$} & \multirow{2}{*}{ Familia } & \multirow{2}{*}{ Especie } & \multicolumn{2}{|c|}{ Ambiente } & \multicolumn{3}{|c|}{ Conservación } \\
\hline & & & BTS & PAS & NOM-059 & UICN & EVS \\
\hline \multirow{17}{*}{$\begin{array}{l}\text { Amphibia } \\
\text { Anura }\end{array}$} & \multirow{2}{*}{ Bufonidae } & Incilius valliceps & $X$ & $X$ & & LC & $6(\mathrm{~B})$ \\
\hline & & Rhinella horribilis & & $\mathrm{X}$ & & $\mathrm{LC}$ & $3(\mathrm{~B})$ \\
\hline & \multirow{2}{*}{ Craugastoridae } & Craugastor alfredi & $X$ & & & VU & $11(\mathrm{M})$ \\
\hline & & Craugastor loki & $\mathrm{X}$ & $X$ & & $\mathrm{LC}$ & $10(\mathrm{M})$ \\
\hline & Eleutherodactylidae & Eleutherodactylus leprus & & $\mathrm{X}$ & & VU & $12(\mathrm{M})$ \\
\hline & \multirow{8}{*}{ Hylidae } & Agalychnis callydrias & $\mathrm{X}$ & $\mathrm{X}$ & & $\mathrm{LC}$ & $11(\mathrm{M})$ \\
\hline & & Dendropsophus ebraccatus & $X$ & $X$ & & $\mathrm{LC}$ & $10(\mathrm{M})$ \\
\hline & & Dendropsophus microcephalus & & $\mathrm{X}$ & & $\mathrm{LC}$ & $7(\mathrm{~B})$ \\
\hline & & Scinax staufferi & $\mathrm{X}$ & $X$ & & $\mathrm{LC}$ & $4(\mathrm{~B})$ \\
\hline & & Smilisca baudinii & $X$ & $X$ & & $\mathrm{LC}$ & $3(\mathrm{~B})$ \\
\hline & & Tlalocohyla loquax & & $X$ & & $\mathrm{LC}$ & $7(\mathrm{~B})$ \\
\hline & & Tlalocohyla picta & $X$ & $X$ & & $\mathrm{LC}$ & $8(\mathrm{~B})$ \\
\hline & & Trachycephalus typhonius & & $X$ & & $\mathrm{LC}$ & $4(\mathrm{~B})$ \\
\hline & \multirow{2}{*}{ Leptodactylidae } & Leptodactylus fragilis & & $X$ & & LC & $5(\mathrm{~B})$ \\
\hline & & Leptodactylus melanonotus & & $X$ & & $\mathrm{LC}$ & $6(\mathrm{~B})$ \\
\hline & \multirow{2}{*}{ Ranidae } & Lithobates brownorum & $\mathrm{X}$ & $\mathrm{X}$ & PR & $\mathrm{NE}$ & $8(\mathrm{~B})$ \\
\hline & & Lithobates vaillanti & $\mathrm{X}$ & $X$ & & $\mathrm{LC}$ & $9(\mathrm{~B})$ \\
\hline \multirow{3}{*}{ Caudata } & \multirow{3}{*}{ Plethodontidae } & Bolitoglossa mexicana & $\mathrm{X}$ & $X$ & PR & $\mathrm{LC}$ & $11(\mathrm{M})$ \\
\hline & & Bolitoglossa platydactyla & $X$ & & PR & NT & $15(\mathrm{M})$ \\
\hline & & Bolitoglossa rufescens & $X$ & $X$ & PR & $\mathrm{LC}$ & $9(\mathrm{~B})$ \\
\hline \multirow{13}{*}{$\begin{array}{l}\text { Reptilia } \\
\text { Squamata } \\
\text { Lacertilia }\end{array}$} & \multirow{2}{*}{ Corytophanidae } & Basiliscus vittatus & & $\mathrm{X}$ & & LC & $7(\mathrm{~B})$ \\
\hline & & Corytophanes hernandesii & $X$ & & PR & $\mathrm{LC}$ & $13(\mathrm{M})$ \\
\hline & \multirow{3}{*}{ Dactyloidae } & Anolis beckeri & $\mathrm{X}$ & & & $\mathrm{NE}$ & $12(\mathrm{M})$ \\
\hline & & Anolis lemurinus & $X$ & $X$ & & $\mathrm{NE}$ & $8(\mathrm{M})$ \\
\hline & & Anolis sericeus & $X$ & $\mathrm{X}$ & & $\mathrm{NE}$ & $8(\mathrm{M})$ \\
\hline & Gekkonidae & Hemidactylus frenatus & & $X$ & & $\mathrm{LC}$ & \\
\hline & \multirow{2}{*}{ Iguanidae } & Iguana iguana & $\mathrm{X}$ & $\mathrm{X}$ & PR & $\mathrm{NE}$ & $12(\mathrm{M})$ \\
\hline & & Ctenosaura acanthura & & $X$ & PR & $\mathrm{NE}$ & $12(\mathrm{M})$ \\
\hline & Phrynosomatidae & Sceloporus variabilis & & $\mathrm{X}$ & & $\mathrm{LC}$ & $5(\mathrm{~B})$ \\
\hline & \multirow{2}{*}{ Scincidae } & Marisora brachypoda & $\mathrm{X}$ & & & $\mathrm{LC}$ & $6(\mathrm{~B})$ \\
\hline & & Scincella cherriei & $X$ & & & $\mathrm{NE}$ & $8(\mathrm{~B})$ \\
\hline & Sphaerodactylidae & Sphaerodactylus glaucus & $\mathrm{X}$ & & PR & $\mathrm{LC}$ & $12(\mathrm{M})$ \\
\hline & Teiidae & Holcosus amphigrammus & $\mathrm{X}$ & & & $\mathrm{NE}$ & \\
\hline \multirow{7}{*}{ Serpentes } & Boidae & Boa imperator & $X$ & $\mathrm{X}$ & & $\mathrm{NE}$ & \\
\hline & \multirow{6}{*}{ Colubridae } & Coluber mentovarius & & $\mathrm{X}$ & A & $\mathrm{LC}$ & $6(\mathrm{~B})$ \\
\hline & & Drymarchon melanurus & $\mathrm{X}$ & & & $\mathrm{LC}$ & $6(\mathrm{~B})$ \\
\hline & & Drymobius margaritiferus & $\mathrm{X}$ & $\mathrm{X}$ & & $\mathrm{NE}$ & $6(\mathrm{~B})$ \\
\hline & & Leptophis mexicanus & $X$ & & & $\mathrm{LC}$ & $6(\mathrm{~B})$ \\
\hline & & Oxybelis aeneus & $X$ & $X$ & & $\mathrm{NE}$ & $5(\mathrm{~B})$ \\
\hline & & Spilotes pullatus & $X$ & & & $\mathrm{NE}$ & $6(\mathrm{~B})$ \\
\hline
\end{tabular}




\begin{tabular}{|c|c|c|c|c|c|c|c|}
\hline \multirow{11}{*}{$\begin{array}{l}\text { Clase } \\
\text { Orden } \\
\text { Suborden }\end{array}$} & \multirow{2}{*}{ Familia } & \multirow{2}{*}{ Especie } & \multicolumn{2}{|c|}{ Ambiente } & \multicolumn{3}{|c|}{ Conservación } \\
\hline & & & BTS & PAS & NOM-059 & UICN & EVS \\
\hline & \multirow{7}{*}{ Dipsadidae } & Coniophanes fissidens & $\mathrm{X}$ & $\mathrm{X}$ & & $\mathrm{NE}$ & $7(\mathrm{~B})$ \\
\hline & & Imantodes cenchoa & $X$ & & PR & $\mathrm{NE}$ & $6(\mathrm{~B})$ \\
\hline & & Leptodeira annulata & & $\mathrm{X}$ & PR & $\mathrm{NE}$ & \\
\hline & & Leptodeira septentrionalis & $\mathrm{X}$ & & & $\mathrm{NE}$ & 8 (B) \\
\hline & & Ninia sebae & $\mathrm{X}$ & $\mathrm{X}$ & & $\mathrm{LC}$ & $5(\mathrm{~B})$ \\
\hline & & Rhadinaea decorata & $\mathrm{X}$ & & & $\mathrm{NE}$ & $9(\mathrm{~B})$ \\
\hline & & Tropidodipsas sartorii & $X$ & & PR & $\mathrm{LC}$ & $9(\mathrm{~B})$ \\
\hline & Leptotyphlopidae & Epictia phenops & $\mathrm{X}$ & & & $\mathrm{NE}$ & \\
\hline & Viperidae & Bothrops asper & $\mathrm{X}$ & $\mathrm{X}$ & & $\mathrm{NE}$ & $12(\mathrm{M})$ \\
\hline \multirow{6}{*}{ Testudines } & Chelydridae & Chelydra rosignonii & $\mathrm{X}$ & & & VU & $17(\mathrm{~A})$ \\
\hline & Emydidae & Trachemys ornata & & $\mathrm{X}$ & & $\mathrm{NE}$ & $19(\mathrm{~A})$ \\
\hline & Geomydidae & Rhinoclemmys areolata & & $\mathrm{X}$ & A & NT & $13(\mathrm{M})$ \\
\hline & \multirow{3}{*}{ Kinosternidae } & Claudius angustatus & & $\mathrm{X}$ & & NT & $14(\mathrm{~A})$ \\
\hline & & Kinosternon leucostomum & & $\mathrm{X}$ & PR & NE & $10(\mathrm{M})$ \\
\hline & & Staurotypus triporcatus & & $\mathrm{X}$ & $\mathrm{A}$ & NT & $14(\mathrm{~A})$ \\
\hline
\end{tabular}

Anfibios

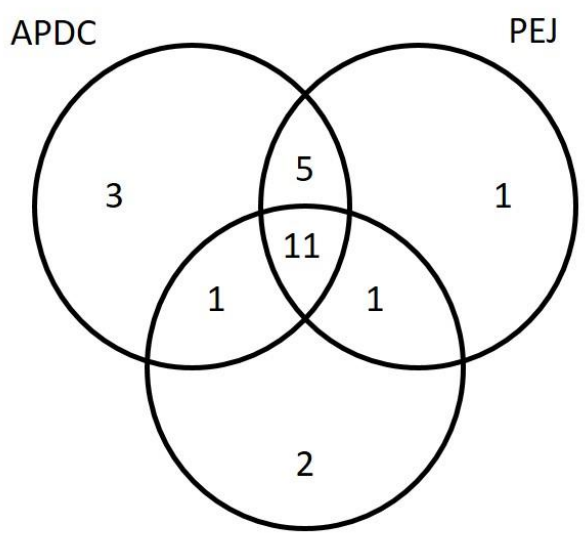

PET
Reptiles

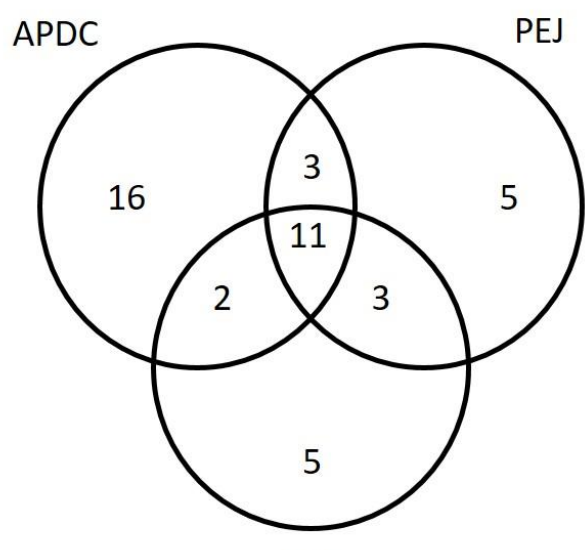

PET

Figura 2. Diagramas de Venn que indican el número de especies exclusivas y compartidas de anfibios y reptiles entre las tres áreas privadas protegidas. Área de Protección y Desarrollo de Ceratozamia (APDC), Parque Ecológico Jaguaroundi (PEJ) y Parque Ecológico Tuzandepetl (PET).

Cuadro 2. Riqueza de especies de anfibios y de reptiles observadas y estimadas en el Área de Protección y Desarrollo de Ceratozamia basadas en métodos no paramétricos. La completitud es un porcentaje de los valores estimados mínimos y máximos con respecto a la riqueza de especies observada.

\section{Estimador de riqueza de especies}

Especies observadas

Chao 2

Jacknife de primer orden

Jacknife de segundo orden

Completitud

\begin{tabular}{cc} 
Anfibios & Reptiles \\
\hline 20 & 35 \\
20 & 44 \\
22 & 47 \\
22 & 50 \\
$91-100$ & $70-79$ \\
\hline
\end{tabular}


Las diferencias en composición de especies entre reservas oscilaron entre el 27 y $47 \%$ en anfibios y entre 46 y $69 \%$ en reptiles. Las mayores diferencias en composición de especies de anfibios se presentaron entre el APDC y PET (47\%) y en los reptiles entre el APDC y PEJ (69\%), mientras que el valor más bajo para anfibios se presentó entre APDC y PEJ (27\%) y para reptiles entre PEJ y PET (46\%). En ambos grupos biológicos, las diferencias en composición estuvieron mayormente explicadas por el reemplazo de especies, con valores entre el 18 y $28 \%$ para anfibios y entre 34 y $38 \%$ para los reptiles (Cuadro 3 ).

En cuanto al estado de conservación de la herpetofauna, el conjunto de las tres reservas alberga tres especies de anfibios y una de reptil incluidas en categorías de riesgo en la UICN y cinco especies de anfibios y 13 de reptiles en la NOM-059-SEMARNAT-2010. El número de especies no evaluadas por la UICN en el conjunto de reservas es de 23.

Cuadro 3. Valores de las diferencias en composición total de especies de anfibios y de reptiles ( $\left.\mathrm{B}_{\mathrm{cc}}\right)$, la proporción debida al reemplazo de especies $\left(\mathrm{B}_{-3}\right)$ y la proporción debida a las diferencias en riqueza de especies $\left(\mathrm{B}_{\text {rich }}\right)$ entre pares de reservas. APDC $=$ Área de Protección y Desarrollo de Ceratozamia, PEJ = Parque Ecológico Jaguaroundi, PET $=$ Parque Ecológico Tuzandepetl.

\begin{tabular}{ccccccc}
\hline & \multicolumn{3}{c}{ Anfibios } & \multicolumn{3}{c}{ Reptiles } \\
\hline Áreas protegidas & $\mathrm{B}_{\mathrm{cc}}$ & $\mathrm{B}_{-3}$ & $\mathrm{~B}_{\text {rich }}$ & $\mathrm{B}_{\mathrm{cc}}$ & $\mathrm{B}_{-3}$ & $\mathrm{~B}_{\text {rich }}$ \\
APDC-PEJ & 0.27 & 0.18 & 0.09 & 0.69 & 0.38 & 0.30 \\
APDC-PET & 0.47 & 0.26 & 0.21 & 0.66 & 0.34 & 0.31 \\
PEJ-PET & 0.42 & 0.28 & 0.14 & 0.46 & 0.38 & 0.07 \\
\hline
\end{tabular}

\section{DISCUSIÓN}

La presencia de las especies en el bosque tropical subcaducifolio o en el pastizal para ganado en el APDC, puede explicarse por los requerimientos ambientales y los hábitos de cada especie, tanto de anfibios como de reptiles (Suazo-Ortuño et al., 2011). En el caso de los anfibios, la presencia de un mayor número de especies en el pastizal para ganado se puede deber a que en este ambiente se ubican cuerpos de agua intermitentes, donde se registraron varias especies como Rhinella horribilis, Dendropsophus microcephalus, Tlalocohyla loquax, Trachycephalus typhonius, Leptodactylus fragilis y L. melanonotus, que se caracterizan por tener una alta tolerancia a la perturbación y por estar asociadas a cuerpos de agua para su reproducción (Lee, 1996; Campbell, 1998). La presencia de un mayor número de especies de reptiles en las zonas de bosque (24 especies), puede deberse a que varias especies de reptiles registrados son de hábitos arborícolas, como Corytophanes hernandesii, Anolis beckeri, Sphaerodactylus glaucus, Imantodes cenchoa, Leptophis mexicanus y Spilotes pullatus (Lee, 1996; Campbell, 1998), lo que restringe su distribución a zonas arboladas.

El APDC, con una extensión de 100 hectáreas (de las cuales sólo la mitad mantiene bosque tropical conservado), alberga una riqueza de especies de anfibios y reptiles mayor a la reportada en las otras dos áreas protegidas privadas de la zona, es decir, en el PEJ, con una extensión casi nueve veces mayor (960 hectáreas), se han registrado 17 especies de anfibios y 24 de reptiles (Herrera et al., 2008); mientras que en el PET, con una extensión 10 veces mayor (1,100 hectáreas) se han registrado 15 especies de anfibios y 22 de reptiles (Cruz-Silva, 2012). Es posible que parte de esta diferencia en el número de especies regis tradas entre las áreas naturales protegidas se deba a las técnicas y esfuerzo de muestreo empleadas en cada estudio (Herrera et al., 2008; Cruz-Silva, 2012). Sin embargo, este mismo patrón de riqueza se ha observado en mamíferos (Ortiz-Lozada et al., 2017), lo que podría indicar que el APDC realmente representa un reservorio de la biodiversidad de los bosques tropicales del sur de México. 


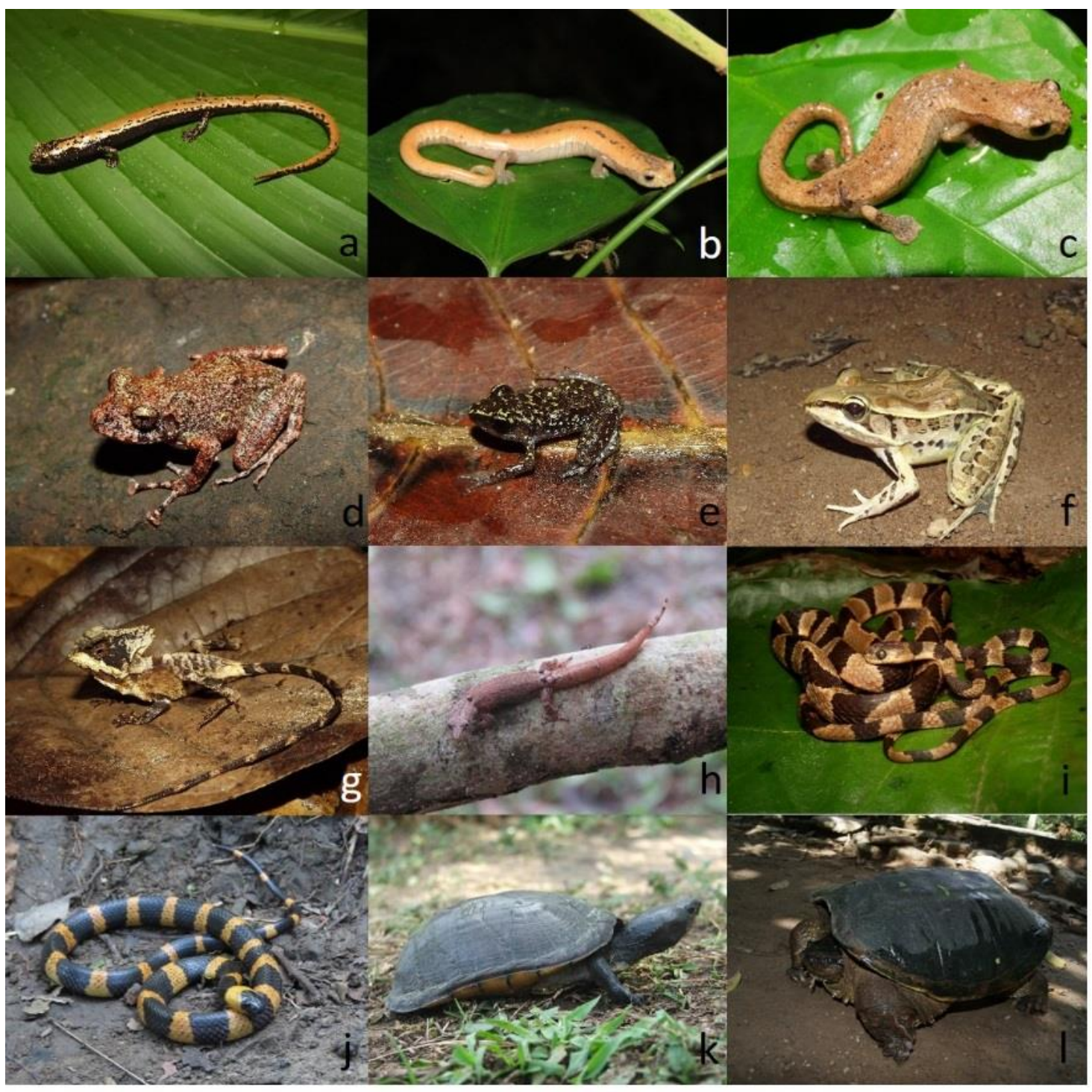

Figura 3. Especies de anfibios y de reptiles registradas en el Área de Protección y Desarrollo de Ceratozamia, en alguna categoría de riesgo de acuerdo con la Unión Internacional para la Conservación de la Naturaleza o la Norma

Oficial Mexicana NOM-059-SEMARNAT-2010 y en categorías de vulnerabilidad ambiental media y alta. a= Bolitoglossa mexicana, $\mathrm{b}=$ Bolitoglossa platydactyla, $\mathrm{c}=$ Bolitoglossa rufescens, $\mathrm{d}=$ Craugastor alfredi, $\mathrm{e}=$ Eleutherodactylus leprus, $\mathrm{f}=$ Lithobates brownorum, $\mathrm{g}=$ Corytophanes hernandesii, $\mathrm{h}=$ Sphaerodactylus glaucus, $\mathrm{i}=$ Imantodes cenchoa, $\mathrm{j}=$ Tropidodipsas sartorii, $\mathrm{k}=$ Kinosternon leucostomum y $\mathrm{l}=$ Chelydra rosignonii .

La riqueza de especies en el APDC podría ser mayor a la informada en el presente estudio, pues los resultados de los cálculos de los estimadores de especies sugieren que el inventario de especies para esta APP es incompleto. De ser así, es necesario incrementar el esfuerzo de muestreo con el fin de registrarlas. Entre las especies de posible presencia en el APDC se encuentran los anfibios Anotheca spinosa, Craugastor pygmaeus, Hypopachus ustus y Gastrophryne elegans, y los reptiles Anolis rodriguezii, A. uniformis, A. tropidonotus, Aspidoscelis deppii, Plestiodon sumichrasti, Clelia scytalina, Mastigodryas melanolomus, Coniophanes imperialis, C. quinquevittatus, Ninia diademata, Micrurus diastema y Crocodylus moreletii. Algunas de estas especies fueron registradas en las otras dos APPs de la zona, en el mismo tipo de vegetación o en otros tipos como encinar-palmar, manglar y popal (Herrera et al., 2008; Cruz-Silva, 2012). Otras especies han sido registradas históricamente en el mismo tipo de vegetación (Aguilar-López \& Canseco- 
Márquez, 2006) o bien en otros tipos de vegetación (Pelcastre-Villafuerte \& Flores-Villela, 1992; Carmona, 2005) en distintos sitios de la región sur del estado de Veracruz.

Nuestros resultados indican que el APDC no alberga una alta proporción de especies de anfibios en elevadas categorías de riesgo de extinción (20\% según la NOM-059, dos especies según la UICN; Fig. 3). En el caso de los reptiles, aunque la proporción de especies amenazadas presentes en el APDC es mayor que en anfibios (una tercera parte (32\%) según la NOM-059-SEMARNAT-2010, una especie según la UICN y $12 \%$ de las especies en categoría de vulnerabilidad ambiental alta; Fig. 3), tampoco podría considerarse alta. Sin embargo, para los reptiles es de resaltar que casi dos tercios (58\%) de las especies presentes en el APDC no han sido evaluadas por la UICN; el registro de estas especies aporta información sobre su distribución y ecología que puede ser útil para que les sea asignada la categoría de riesgo de extinción más adecuada.

Las diferencias en composición de herpetozoos entre las tres reservas (APDC, PEJ, PET) muestran escenarios distintos para anfibios y reptiles. Por un lado, los anfibios tienen una composición de especies relativamente similar (valores de $\mathrm{B}_{\mathrm{cc}}$ entre 0.27 y 0.48 ), mientras que la composición de reptiles tiende a ser distinta (valores de $\mathrm{B}_{\mathrm{cc}}$ entre 0.46 y 0.69). Las diferencias en composición en ambos grupos pueden deberse a que cada reserva tiene algún tipo de vegetación distinto, como en el caso de PEJ, donde hay áreas cubiertas por bosque tropical perennifolio (Herrera et al., 2008) o en el caso de PET, que tiene áreas cubiertas por vegetación halófila, zonas de palmar, manglar y vegetación inundable (Cruz-Silva, 2012). En el caso de los reptiles, las diferencias en composición necesitan ser consideradas con precaución, puesto que es posible que los valores obtenidos se deban, en parte, a los bajos niveles de completitud (por debajo del 80\%) en el inventario de especies en el APDC.

Nuestros resultados indican que por la riqueza de especies que alberga, las especies amenazadas de extinción y el número de especies (principalmente de reptiles) aún no evaluadas por la UICN, el APDC contribuye a la conservación de poblaciones de anfibios y reptiles en una zona altamente transformada del sur de México, en conjunto con las otra dos APPs, donde no existen otras áreas naturales protegidas (Bezaury-Creel et al., 2011) que garanticen la conservación de los herpetozoos (Urbina-Cardona \& FloresVillela, 2010). En conjunto, las tres reservas albergan el 53\% (24 especies) de los 45 anfibios y el $43 \%$ (47 especies) de los 108 reptiles registrados en la región sur del estado de Veracruz (Pelcastre-Villafuerte \& Flores-Villela, 1992) y el $20 \%$ de las 120 especies anfibios y el $17.5 \%$ de las 270 especies de reptiles registradas para el sureste de México (Johnson et al., 2010). El APDC y las otras dos APPs complementan la función de conservación biológica de la Reserva de la Biosfera de Los Tuxtlas bajo gobernanza gubernamental, localizada a $50 \mathrm{~km}$ en línea recta, pues aunque casi la totalidad de las especies de herpetozoos presentes en las tres reservas (excepto Bolitoglossa mexicana y Sceloporus variabilis) han sido registradas en Los Tuxtlas (López-Luna, 2017), su presencia en las tres APPs permite garantizar la permanencia de poblaciones en distintos puntos de su distribución.

Para garantizar el mantenimiento del mayor número de especies de anfibios y de reptiles, y potencialmente de otros grupos biológicos en la zona donde se ubican el APDC y las otras dos reservas privadas, es necesario implementar actividades que han probado ser efectivas en la conservación de anfibios y reptiles en paisajes modificados. Por ejemplo 1) la restauración ecológica de bosques que fueron transformados en pastizales inducidos y otros ambientes modificados (Smith et al., 2015; Díaz-García et al., 2017), 2) la protección de los bosques ribereños (Rodríguez-Mendoza \& Pineda, 2010), 3) el establecimiento de cercas vivas (Pérez, 2002; Harvey et al., 2005) que ayudan al movimiento de algunas especies a través del paisaje y 4) proteger las zonas de vegetación secundaria en proceso de sucesión (Gillespie et al., 2012). Finalmente, se sugiere implementar programas de educación ambiental, con énfasis en la protección de las serpientes, las cuales son exterminadas por miedo (Wojnowski, 2010); así como de tortugas e iguanas que son consumidas con fines alimenticios (Morales-Mávil \& Villa-Cañedo, 1998). 
AgRAdECIMIENTOS. Agradecemos a Braskem Idesa por el financiamiento para la elaboración de este estudio, especialmente a Roberto Velasco (Director Industrial Complejo), Antonio Santos Souza Galvão (Sustentabilidad) y Ana Luisa Martínez López (Medio Ambiente). A José Alberto Toto, David Antonio Suárez y Sergio Trinidad Vandyck Alemán, por su apoyo en el trabajo de campo. Agradecemos a Erasmo Cázares Hernández por la foto de Kinosternon leucostomum y a Cynthia Ramírez González por la fotografía de Chelydra rosignonii. Agradecemos a Raziel Lucio, Georgina Santos y Ricardo Luría por sus valiosos comentarios al manuscrito.

\section{LITERATURA CITADA}

Aguilar-López, J. L., Canseco-Márquez, L. (2006) Herpetofauna del municipio de Las Choapas, Veracruz, México. Boletín de la Sociedad Herpetológica Mexicana, 14, 20-37.

Bezaury-Creel, J. E., Torres, J. F., Ochoa-Ochoa, L. M., Castro-Campos, M. (2011) Áreas naturales protegidas y otros espacios destinados a la conservación, restauración y uso sustentable de la biodiversidad en México-2011 (Formato Mapa-Cartel). The Nature Conservancy-México.

Bezaury-Creel, J. E. (2014) Mexico. Pp. 80-83. En: S. Stolton, K. H. Redford, N. Dudley. The futures of Privately Protected Areas. Gland, Switzerland. IUCN.

Böhm, M., Collen, B., Baillie, J. E. M., Bowles, P., Chanson, J., Cox, N., Hammerson, G., Hoffmann, M., Livingstone, S. R., Ram, M., Rhodin, A. G. J., Stuart, S. N., Van Dijk, P. P., Young, B. E., Afuang, L. E., Aghasyan, A., García, A., Aguilar, C., Ajtic, R., Akarsu, F., Alencar, L. R. V., Allison, A., Ananjeva, N., Anderson, S., Andrén, C., Ariano-Sánchez, D., Arredondo, J. C., Auliya, M., Austin, C. C., Avci, A., Baker, P. J., Barreto-Lima, A. F., Barrio-Amorós, C. L., Basu, D., Bates, M. F., Batistella, A., Bauer, A., Bennett, D., Böhme, W., Broadley, D., Brown, R., Burgess, J., Captain, A., Carreira, S., Castaneda, M. D. R., Castro, F., Catenazzi, A., Cedeno-Vázquez, J. R., Chapple, D. G., Cheylan, M., Cisneros Heredia, D. F., Cogalniceanu, D., Cogger, H., Corti, C., Costa, G. C., Couper, P. J., Courtney, T., Crnobrnja-Isailovic, J., Crochet, P. A., Crother, B., Cruz, F., Daltry, J. C., Daniels, R. J. R., Das, I., de Silva, A., Diesmos, A. C., Dirksen, L., Doan, T. M., Dodd, C. K., Doody, J. S., Dorcas, M. E., Duarte de Barros Filho, J., Egan, V. T., El Mouden, E. H., Embert, D., Espinoza, R. E., Fallabrino, A., Feng, X., Feng, Z. J., Fitzgerald L., Flores-Villela, O., França, F. G. R., Frost, D., Gadsden, H., Gamble, T, Ganesh, S. R., Garcia, M. A., García-Pérez, J. E., Gatus, J., Gaulke, M., Geniez, P., Georges, A., Gerlach, J., Goldberg, S., Gonzalez, J. C. T., Gower, D. J., Grant, T., Greenbaum, E., Grieco, C., Guo, P., Hamilton, A. M., Hare, K., Hedges, S. B., Heideman, N., Hilton-Taylor, C., Hitchmough, R., Hollingsworth, B., Hutchinson, M., Ineich, I., Iverson, J., Jaksic, F. M., Jenkins, R., Joger, U., Jose, R., Kaska, Y., Kaya, U., Keogh, J. S., Köhler, G., Kuchling, G., Kumlutaş, Y., Kwet, A., La Marca, E., Lamar, W., Lane, A., Lardner, B., Latta, C., Latta, G, Lau, M., Lavin, P., Lawson, D., LeBreton, M., Lehr, E., Limpus, D., Lipczynski, N., Lobo, A. S., López-Luna, M. A., Luiselli, L., Lukoschek, V., Lundberg, M., Lymberakis, P., Macey, R., Magnusson, W. E., Mahler, D. L., Malhotra, A., Mariaux, J., Maritz, B., Marques, O. A. V., Márquez, R., Martins, M., Masterson, G., Mateo, J. A., Mathew, R., Mathews, N., Mayer, G., McCranie, J. R., Measey, G. J., Mendoza-Quijano, F., Menegon, M., Métrailler, S., Milton, D. A., Montgomery, C., Morato, S. A. A., Mott, T., Munoz-Alonso, A., Murphy, J., Nguyen, T. Q., Nilson, G., Nogueira, C., Núnez, H., Orlov, N., Ota, H., Ottenwalder, J., Papenfuss, T., Pasachnik, S., Passos, P., Pauwels, O. S. G., Pérez-Buitrago, N., Pérez-Mellado, V., Pianka, E. R., Pleguezuelos, J., Pollock, C., Ponce-Campos, P., Powell, R., Pupin, F., Quintero Díaz, G. E., Radder, R., Ramer, J., Rasmussen, A. R., Raxworthy, C., Reynolds, R., Richman, N., Rico, E. L., Riservato, E., Rivas, G., da Rocha, P. L. B., Rödel, M. O., Rodríguez Schettino, L., Roosenburg, W. M., Ross, J. P., Sadek, R., Sanders, K., SantosBarrera, G., Schleich, H. H., Schmidt, B. R., Schmitz, A., Sharifi, M., Shea, G., Shi, H. T., Shine, R., Sindaco, R., Slimani, T., Somaweera, R., Spawls, S., Stafford, P., Stuebing, R., 
Sweet, S., Sy, E., Temple, H. J., Tognelli, M. F., Tolley, K., Tolson, P. J., Tuniyev, B., Tuniyev, S., üzüm, N., van Buurt, G., Van Sluys, M., Velasco, A., Vences, M., Veselý, M., Vinke, S., Vinke, T., Vogel, G., Vogrin, M., Vogt, R. C., Wearn, O. R., Werner, Y. L., Whiting, M. J., Wiewandt, T., Wilkinson, J., Wilson, B., Wren, S., Zamin, T., Zhou, K. \& Zug, G. (2013) The conservation status of the world's reptiles. Biological Conservation, 157, 372-385.

Campbell, J. A. (1998) Amphibians and reptiles of Northern Guatemala, the Yucatán, and Belize. University of Oklahoma Press, Oklahoma, USA, $380 \mathrm{pp}$.

Carmona, F. (2005) Diversidad herpetofaunística de un remanente de la selva alta perennifolia al sur de Veracruz y su afinidad con zonas cercanas. Tesis de Licenciatura. Facultad de Ciencias, Universidad Nacional Autónoma de México, 117 pp.

Carvalho, J. C., Cardoso, P., Gomes, P. (2012) Determining the relative roles of species replacement and species richness differences in generating beta-diversity patterns. Global Ecology and Biogeography, 21, 760-771.

Castillo-Campos, G., Avendaño-Reyes, S., Medina-Abreo, M. E. (2011) Flora y vegetación. Pp. 163180. En: Comisión Nacional para el Conocimiento y Uso de La Biodiversidad (CONABIO). La biodiversidad en Veracruz: estudio de estado. CONABIO, Gobierno del Estado de Veracruz, Universidad Veracruzana, Instituto de Ecología A. C. México.

Colwell, R. K. (2013) EstimateS: statistical estimation of species richness and shared species from samples. Versión 9.1 Disponible en línea: http://purl.oclc.org/estimates

CONANP (Comisión Nacional de Áreas Naturales Protegidas) (2006) Programa de Conservación y Manejo Reserva de la Biosfera Los Tuxtlas. México, 293 pp.

Cortes-Gómez, A. M., Ruiz-Agudelo, C. A., Valencia-Aguilar, A., Ladle, R. J. (2015) Ecological functions of neotropical amphibians and reptiles: a review. Universitas Scientiarum, 20, 229-245.

Crump, M. L., Scott Jr., N. J. (1994) Visual encounter surveys. Pp. 84-92. En: R. W. Heyer, M. A. Donelly, R. W. McDiarmid, L. C. Hayek, M. S. Foster (Eds.). Measuring and monitoring biological diversity: standard methods for amphibians. Smithsonian Institution Press, USA.

Cruz-Bazán, E. J., Pech-Canché, J. M., Cimé-Pool, J. A. (2017) Diversidad de mamíferos terrestres en un área privada de conservación en México. Ecosistemas y Recursos Agropecuarios, 4, 123-133.

Cruz-Elizalde, R., Ramírez-Bautista, A., Wilson, L. D., Hernández-Salinas, U. (2015) Effectiveness of protected areas in herpetofaunal conservation in Hidalgo, Mexico. Herpetological Journal, 25, 4148.

Cruz-Silva, A. (2012) Anfibios y Reptiles. Pp. 20-41. En: UNAM. Estudios técnicos para definir el desarrollo y funcionamiento del Parque Ecológico Tuzandepetl. México. Instituto de Biología, UNAM.

Díaz-García, J. M., Pineda, E., López-Barrera, F., Moreno, C. E. (2017) Amphibian species and functional diversity as indicators of restoration success in tropical montane forest. Biodiversity and Conservation, 26, 2569-2589.

Frost, D. R. (2017) Amphibian Species of the World: An Online Reference. Version 6.0. Disponible en línea: http://research.amnh.org/herpetology/amphibia/index.html. American Museum of Natural History, New York, USA. (última consulta el 15 de junio 2017).

Gillespie, G. R., Ahmad, E., Elahan, B., Evans, A., Ancrenaz, M., Goossens, B., Scroggie, M. P. (2012) Conservation of amphibians in Borneo: Relative value of secondary tropical forest and non-forest habitats. Biological Conservation, 152, 136-144.

Hansen, M. C., Potapov, P. V., Moore, R., Hancher, M., Turubanova, S. A., Tyukavina, A., Thau, D., Stehman, S. V., Goetz, S. J., Loveland, T. R., Kommareddy, A., Egorov, A., Chini, L., Justice, C. O., Townshend, J. R. G. (2013) High-resolution global maps of 21st-century forest cover change. Science, $342,850$.

Harvey, C. A., Villanueva, C., Villacís, J., Chacón, M., Muñoz, D., López, M., Ibrahim, M., Gómez, R., Taylor, R., Martinez, J., Navas, A., Saenz, J., Sánchez, D., Medina, A., Vichez, S., Hernández, B., Perez, A., Ruiz, F., López, F., Lang, I., Sinclair, F. L. (2005) Contribution of 
live fences to the ecological integrity of agricultural landscapes. Agriculture Ecosystems and Environment, 111, 200-230.

Herrera, G. L., Reynoso, V. H., Curiel, D., Ramírez, N., Rodríguez, M., Mirón, L., Sánchez, R., Aguilar, S., Carmona, F., Urbina, J., González, A. (2008) La riqueza faunística en un ambiente perturbado: El caso del Parque Ecológico Jaguaroundi. Pp.79-100. En: Y. Nava, I. Rosas (Coords.). El parque ecológico Jaguaroundi, conservación de la selva veracruzana en una zona industrializada. SEMARNAT, INE, UNAM, PEMEX, México.

IUCN (International Union for Conservation of Nature) (2017) The IUCN Red List of Threatened Species. Version 2017-1. Disponible en línea: http://www.iucnredlist.org (última consulta el 12 de mayo 2017).

Johnson, J. D., Mata-Silva, V., Ramírez-Bautista, A. (2010) The herpetofauna of southeastern Mexico: biogeography and conservation. Pp. 322-369. En: L. D. Wilson, J. H. Townsend, J. Johnson (Eds.). Conservation of Mesoamerican amphibians and reptiles. Eagle Mountain Publishing, Utah, USA.

Köhler, G. (2008) Reptiles of Central America. Herpeton, Offenbach, Germany, 367 pp.

Köhler, G. (2011) Amphibians of Central America. Herpeton, Offenbach, Germany, 379 pp.

Lee, J. C. (1996) The amphibians and reptiles of the Yucatan Peninsula. Cornell University Press, New York, $500 \mathrm{pp}$.

López-Luna, M. A. (2017) Estado actual de la diversidad y poblaciones de los anfibios y reptiles en Los Tuxtlas. Pp. 347-369. En: V. H. Reynoso, R. I. Coates, M. L. Vázquez-Cruz (Eds.). Avances y Perspectivas en la Investigación de los Bosques Tropicales y sus Alrededores: La Región de Los Tuxltas. Instituto de Biología, Universidad Nacional Autónoma de México, Ciudad de México.

Morales-Mávil, J. E., Villa-Cañedo, J. T. (1998) Notas sobre el uso de la fauna Silvestre en Catemaco, Veracruz, México. Acta Zoológica Mexicana (nueva serie), 73, 127-143.

Moreno, C. E. (2001) Métodos para medir la biodiversidad. Vol. 1. Zaragoza: M\&T-Manuales y Tesis SEA, $83 \mathrm{pp}$.

Naughton-Treves, L., Buck, H. M., Brandon, K. (2005) The role of protected areas in conserving biodiversity and sustaining local livelihoods. Annual Review of Environment and Resources, 30: 219-252.

Ochoa-Ochoa, L., Urbina-Cardona, J. N., Vázquez, L. B., Flores-Villela, O., Bezaury-Creel, J. (2009) The effects of governmental protected areas and social initiatives for land protection on the conservation of Mexican amphibians. PLoS ONE, 4, e6878.

Ortiz-Lozada, L., Pelayo-Martínez, J., Mota-Vargas, C., Demeneghi-Calatayud, A. P., Sosa, V. J. (2017) Absence of large and presence of medium-sized mammal species of conservation concern in a privately protected area of rain forest in southern Mexico. Tropical Conservation Science, 10, $1-13$.

Pelcastre-Villafuerte, L., Flores-Villela, O. (1992) Lista de especies y localidades de recolecta de la herpetofauna de Veracruz, México. Publicaciones Especiales del Museo de Zoología, 4, 25-96.

Pérez, A. M. (2002) Redes ecológicas: un uso alternativo del término y su aplicación a la conservación de la diversidad in situ. Un enfoque preliminar. Gaia, 2, 1-10.

Rodríguez-Mendoza, C., Pineda, E. (2010) Importance of riparian remnants for frog species diversity in a highly fragmented rainforest. Biology Letters, 6, 781-784.

Sánchez-Colón, S., Flores-Martínez, A., Cruz-Leyva, I. A., Velázquez, A. (2009) Estado y transformación de los ecosistemas terrestres por causas humanas. Pp. 75-129. En: CONABIO. Capital natural de México, estado de conservación y tendencias de cambio (vol. II). Comisión Nacional para el Conocimiento y Uso de la Biodiversidad, México.

Schmitter-Soto, J. J., Mariaca-Méndez, R., Soto-Pinto, M. L. (2016) Una breve historia del conocimiento y uso de la biodiversidad en la frontera sur de México. Sociedad y Ambiente, 4, 160173.

SEDEMA (Secretaría de Medio Ambiente del Estado de Veracruz) (2015) Certificado SEDEMA/DGGARN-APC/11/215 y SEDEMA/DGGARN-APC/12/215. Emitida 30 de noviembre 2015. 
SEMARNAT (Secretaría de Medio Ambiente y Recursos Naturales) (2010) Norma Oficial Mexicana NOM-059. Protección ambiental de especies nativas de México de flora y fauna silvestresCategorías de riesgo y especificaciones para su inclusión, exclusión o cambio-Lista de especies en riesgo. Proyecto de Modificación. Diario oficial de la Federación. Diciembre 2015, México.

Smith, G. C., Lewis, T., Hogan, L. D. (2015) Fauna community trends during early restoration of alluvial open forest/Woodland ecosystems on former agricultural land. Restoration Ecology, 23, 787-799.

Sommer, C. I., Oropeza-Orozco, O. (2010) Atlas regional de impactos derivados de las actividades petroleras en Coatzacoalcos, Veracruz. SEMARNAT, UNAM, INE, Instituto de Geografía, México, $119 \mathrm{pp}$.

Stolton, S., Redford, H. K., Dudley, N. (2014) The Futures of Privately Protected Areas. IUCN. Gland, Switzerland, $111 \mathrm{pp}$.

Suazo-Ortuño, I., Alvarado-Díaz, J., Martínez-Ramos, M. (2011) Riparian areas and conservation of herpetofauna in a tropical dry forest in western Mexico. Biotropica, 43, 237-24.

Uetz, P., Freed, P., Hosek, J. (Eds). The Reptile Database. Disponible en línea: http://www.reptiledatabase.org (última consulta el 3 de enero 2018).

Urbina-Cardona, J. N., Flores-Villela, O. (2010) Ecological-niche modeling and prioritization of conservation-area networks for Mexican herpetofauna. Conservation Biology, 24, 1031-1041.

Wake, D. B. (1991) Declining amphibian populations. Science, 253, 860.

Wilson, L. D., Mata-Silva, V., Johnson, J. D. (2013a) A conservation reassessment of the amphibians of Mexico based on the EVS measure. Amphibian and Reptile Conservation, 7, 97-127.

Wilson, L. D., Mata-Silva, V., Johnson, J. D. (2013b) A conservation reassessment of the reptiles of Mexico based on the EVS measure. Amphibian and Reptile Conservation, 7, 1-47.

Wojnowski, D. (2010) Gender-appropriate responses to snakes in Kenya: culture, concepts and context. The International Journal of Interdisciplinary Social Sciences, 5, 1-12. 